Dariusz Banaszewski, Mathematics Department, Pedagogical University, Chodkiewicza 30, 85-064 Bydgoszcz, Poland.

\title{
UNIVERSALLY BAD DARBOUX FUNCTIONS IN THE CLASS OF ADDITIVE FUNCTIONS
}

\begin{abstract}
The main result: For every family $\mathcal{G}$ of additive functions with card $\mathcal{G}=2^{\omega}$ if the covering of the family of all level sets of functions from $\mathcal{G}$ is equal to $2^{\omega}$, then there exists an additive Darboux function $f$ such that $f+g$ is Darboux for no $g \in \mathcal{G}$.
\end{abstract}

Definitions. Let us establish some terminology to be used. For a subset $A$ of $\mathbb{R} \times \mathbb{R}$ we denote by $\operatorname{dom}(A)$ and $\operatorname{rng}(A)$ the $x$-projection and $y$-projection of $A$. We say that $f: \mathbb{R} \rightarrow \mathbb{R}$ is a Darboux function whenever $f(J)$ is connected for every interval $J \subset \mathbb{R}$. The family of all such functions we will denote by $\mathcal{D}$.

We shall consider $\mathbb{R}$ as a linear space over $\mathbb{Q}$, the set of rationals. Every base of this space will be referred to as a Hamel basis. It is evident that the cardinality of every Hamel basis is equal to $2^{\omega}$.

If $A \subset \mathbb{R}$ is an arbitrary nonempty set, then by $L(A)$ we mean the linear subspace of $\mathbb{R}$ spanned over $A$, i.e., the set of all finite linear combinations of elements of $A$ (with coefficients from $\mathbb{Q}$ ). Analogously, for an arbitrary nonempty planar set $A \subset \mathbb{R} \times \mathbb{R}$ we put the set $L_{2}(A)$. For any $A \subset \mathbb{R}$ and $x \in \mathbb{R}$ we define $x+A=\{x+a: a \in A\}$.

Let $L$ be a linear subspace of $\mathbb{R}$ over $\mathbb{Q}$. A function $f: L \rightarrow \mathbb{R}$ is called additive iff it satisfies Cauchy's equation $f(x+y)=f(x)+f(y)$ for all $x, y \in L$ [2]. (See also [5, p. 120], for the history of this notion.) Recall that every additive function $f: \mathbb{R} \rightarrow \mathbb{R}$ can be obtained as the unique additive extension of a function defined on a Hamel basis. The class of all additive functions from $\mathbb{R}$ to $\mathbb{R}$ will be denoted by $\mathcal{A} d d$.

Key Words: Darboux function, additive function, universally bad Darboux function, maximal additive family

Mathematical Reviews subject classification: Primary 26A15. Secondary 26A51

Received by the editors May 15, 1996 
We do not distinguish between a function $f: L \rightarrow \mathbb{R}$ where $L \subset \mathbb{R}$ and its graph (i.e., a subset of $\mathbb{R} \times \mathbb{R}$ ). By $f+g$ we mean the function $h: \operatorname{dom}(f) \cap$ $\operatorname{dom}(g) \rightarrow \mathbb{R}$ such that $h(x)=f(x)+g(x)$ for each $x \in \operatorname{dom}(f) \cap \operatorname{dom}(g)$.

The cardinality of a set $A$ is denote by card $(A)$. Cardinals are identified with initial ordinals. For an arbitrary cover $\mathcal{B}$ of the real line we define the covering of $\mathcal{B}$ as the smallest cardinal $\kappa$ for which there exists a subfamily $\mathcal{B}_{0} \subset \mathcal{B}$ with card $\left(\mathcal{B}_{0}\right)=\kappa$ and $\mathbb{R}=\bigcup \mathcal{B}_{0}$.

For a family $\mathcal{F} \subset \mathbb{R}^{\mathbb{R}}$ we denote by $\mathcal{M}_{a}(\mathcal{F})$ the maximal additive family for $\mathcal{F}$, i.e.,

$$
\mathcal{M}_{a}(\mathcal{F})=\left\{g \in \mathbb{R}^{\mathbb{R}}: f+g \in \mathcal{F} \text { for each } f \in \mathcal{F}\right\} \text {. }
$$

Recall that $\mathcal{M}_{a}(\mathcal{D})$ is equal to the family of all constant functions [6].

For an infinite cardinal $\kappa$ we say that a cardinal number $\lambda$ is the cofinality of $\kappa$ (and write $\lambda=\mathrm{cf}(\kappa))$ if $\lambda$ is the least cardinal number such that there exists a family of sets $\left(X_{i}\right)_{i \in \lambda}$ with the property that $\bigcup_{i \in \lambda} X_{i}=\kappa$ and $\operatorname{card}\left(X_{i}\right)<\kappa$ for every $i \in \lambda$. For cardinals $\kappa$ we say that $\kappa$ is a regular cardinal if $\kappa=\operatorname{cf}(\kappa)$.

Given a family $\mathcal{F} \subset \mathbb{R}^{\mathbb{R}}$ consider the condition

$c(\mathcal{F})$ : there is a $f \in \mathcal{D}$ such that $f+g \notin \mathcal{D}$ for each $g \in \mathcal{F}$.

Such a function $f$ is called a universally bad Darboux function for $\mathcal{F}$. Determining for which families $\mathcal{F}$ the condition $c(\mathcal{F})$ is fulfilled is a problem considered by several authors (see, e.g., [6], [8], [1], [3] and [4]). In particular, if the additivity of the ideal of all first category subsets of $\mathbb{R}$ is equal to $2^{\omega}$ (e.g., if Martin's Axiom or $\mathrm{CH}$ hold), then $c\left(C^{*}\right)$ holds for the family $C^{*}$ of all nowhere constant, continuous functions [3]. On the other hand, there is a model of set theory in which $c\left(C^{*}\right)$ fails to hold. ([7]) In this paper we study analogous problems for the class of additive functions.

Lemma 1 Let $f \in \mathcal{A}$ dd be such that $\operatorname{ker}(f) \neq\{0\}$ and $\operatorname{rng}(f)=\mathbb{R}$. Then $f$ has the Darboux property.

Proof. Observe that $f \in \mathcal{A} d d, \operatorname{ker}(f)$ is a linear subspace of $\mathbb{R}$ and, since $\operatorname{ker}(f) \neq\{0\}, \operatorname{ker}(f)$ is dense in $\mathbb{R}$. Moreover, it is well-known that each two level sets of an additive function are congruent under translations, so any level set of $f$ is dense in $\mathbb{R}$. Hence $f(I)=\mathbb{R}$ for every interval $I \subset \mathbb{R}$ and $f \in \mathcal{D}$.

Lemma 2 Let $\mathcal{B}$ be a cover of $\mathbb{R}$ such that card $(\mathcal{B})=2^{\omega}$ and the covering of $\mathcal{B}$ is equal to $2^{\omega}$. There exists a Hamel basis $H$ such that $H \backslash \cup \mathcal{B}^{*} \neq \emptyset$ for every $\mathcal{B}^{*} \subset \mathcal{B}$ with $\operatorname{card}\left(\mathcal{B}^{*}\right)<\operatorname{cf}\left(2^{\omega}\right)$. 
Proof. Let $\mathcal{B}=\left\{B_{\alpha}: \alpha<2^{\omega}\right\}$ and $h_{0} \neq 0$. Fix $\alpha<2^{\omega}$ and assume that we have chosen a linearly independent set $\left\{h_{\beta}: \beta<\alpha\right\}$ such that $h_{\beta} \notin \bigcup_{\gamma<\beta} B_{\gamma}$ for each $\beta<\alpha$. Let $E_{\alpha}=L\left(\left\{h_{\beta}: \beta<\alpha\right\}\right)$. For each $x \in E_{\alpha}$ choose $C_{x} \in \mathcal{B}$ with $x \in C_{x}$. Since card $\left(E_{\alpha}\right)<2^{\omega}$, by our assumption we obtain that the family $\mathcal{B}_{\alpha}=\left\{C_{x}: x \in E_{\alpha}\right\} \cup\left\{B_{\beta}: \beta<\alpha\right\}$ does not cover $\mathbb{R}$. Choose $h_{\alpha} \in \mathbb{R} \backslash \bigcup \mathcal{B}_{\alpha}$. Then the set $\left\{h_{\alpha}: \alpha<2^{\omega}\right\}$ is linearly independent. Let $H$ be a Hamel basis containing $\left\{h_{\alpha}: \alpha<2^{\omega}\right\}$. For every $\mathcal{B}^{*} \subset \mathcal{B}$ with card $\left(\mathcal{B}^{*}\right)<$ $\operatorname{cf}\left(2^{\omega}\right)$ there is $\alpha<2^{\omega}$ such that $\mathcal{B}^{*} \subset\left\{B_{\beta}: \beta<\alpha\right\}$, so $h_{\alpha} \in H \backslash \mathcal{B}^{*}$.

If we assume that $2^{\omega}$ is a regular cardinal, then for each cover $\mathcal{B}$ of $\mathbb{R}$ such that the covering of $\mathcal{B}$ equals $2^{\omega}$ there exists a Hamel basis $H$ such that $H \backslash \bigcup \mathcal{B}^{*} \neq \emptyset$ for every $\mathcal{B}^{*} \subset \mathcal{B}$ with card $\left(\mathcal{B}^{*}\right)<2^{\omega}$. We are unable to determine whether this statement can be proved in ZFC.

Theorem 1 Assume that $2^{\omega}$ is a regular cardinal and $\mathcal{G}=\left\{g_{\alpha}: \alpha<2^{\omega}\right\} \subset$ Add satisfies the condition

$(\star)$ the covering of the family $\mathcal{B}=\left\{g^{-1}(y): g \in \mathcal{G} \& y \in \mathbb{R}\right\}$ is equal to $2^{\omega}$.

Then there is $f \in \mathcal{A} d d \cap \mathcal{D}$ such that $f+g_{\alpha} \notin \mathcal{D}$ for each $\alpha<2^{\omega}$.

Proof. Let $H=\left\{h_{\alpha}: \alpha<2^{\omega}\right\}$ be the Hamel basis constructed in Lemma 2 for the cover $\mathcal{B}$. For every $\alpha<2^{\omega}$ we will construct an additive function $f_{\alpha}$ and choose $u_{\alpha} \in H$ such that

(i) $f_{\beta} \subset f_{\alpha}$ for $\beta<\alpha$,

(ii) $h_{\alpha} \in \operatorname{dom}\left(f_{\alpha}\right) \cap \operatorname{rng}\left(f_{\alpha}\right)$ and $\operatorname{ker}\left(f_{\alpha}\right) \neq\{0\}$,

(iii) $\operatorname{rng}\left(f_{\alpha}+g_{\alpha}\right) \neq\{0\}$,

(iv) $u_{\beta} \notin \operatorname{rng}\left(f_{\alpha}+g_{\beta}\right)$ for each $\beta \leq \alpha$,

(v) $\operatorname{card}\left(\operatorname{dom}\left(f_{\alpha}\right)\right)=\max (\omega, \operatorname{card}(\alpha))$.

First assume that $\alpha=0$. Choose $h \in H$ such that $h \neq-g_{0}\left(h_{2}\right)$ and set $f_{0}=L_{2}\left(\left\{\left(h_{0}, 0\right),\left(h_{1}, h_{0}\right),\left(h_{2}, h\right)\right\}\right)$. Since card $\left(\operatorname{rng}\left(f_{0}+g_{0}\right)\right)=\omega$, we can choose $u_{0} \in H \backslash \operatorname{rng}\left(f_{0}+g_{0}\right)$. It is easy to verify that $f_{0}$ and $u_{0}$ fulfill conditions (i) $-(\mathrm{v})$.

Now fix $\alpha<2^{\omega}$ and assume that we have chosen for $\beta<\alpha$ functions $f_{\beta}$ and points $u_{\beta}$ which satisfy conditions (i) $-(\mathrm{v})$. Set $f_{\alpha}^{(0)}=\bigcup_{\beta<\alpha} f_{\beta}$. We consider two cases. If $h_{\alpha} \in \operatorname{rng}\left(f_{\alpha}^{(0)}\right)$, then define $f_{\alpha}^{(1)}=f_{\alpha}^{(0)}$. Otherwise we will choose $y_{\alpha} \in H$ such that $y_{\alpha} \notin \operatorname{dom}\left(f_{\alpha}^{(0)}\right)$ and

$$
u_{\beta} \notin L\left(\operatorname{rng}\left(f_{\alpha}^{(0)}+g_{\beta}\right) \cup\left\{g_{\beta}\left(y_{\alpha}\right)+h_{\alpha}\right\}\right) \text { for } \beta<\alpha .
$$


To choose $y_{\alpha}$ observe that the family

$$
\mathcal{B}_{\alpha}=\bigcup_{\beta<\alpha}\left\{g_{\beta}^{-1}(y): y \in L\left(\operatorname{rng}\left(f_{\alpha}^{(0)}+g_{\beta}\right) \cup\left\{u_{\beta}\right\}\right)-h_{\alpha}\right\} \cup\left\{g_{\alpha}^{-1}\left(h_{\alpha}\right)\right\}
$$

has cardinality less than $2^{\omega}$. For each $x \in \operatorname{dom}\left(f_{\alpha}^{(0)}\right)$ choose $C_{x} \in \mathcal{B}$ with $x \in C_{x}$. Since card $\left(\operatorname{dom}\left(f_{\alpha}^{(0)}\right)\right)<2^{\omega}, H \backslash\left(\cup \mathcal{B}_{\alpha} \cup \cup_{x \in \operatorname{dom}\left(f_{\alpha}^{(0)}\right)} C_{x}\right) \neq \emptyset$. Take an arbitrary

$$
y_{\alpha} \in H \backslash\left(\bigcup \mathcal{B}_{\alpha} \cup \bigcup_{x \in \operatorname{dom}\left(f_{\alpha}^{(0)}\right)} C_{x}\right)
$$

To prove (1) fix $\beta<\alpha$ and suppose that $u_{\beta}=q_{1} y+q_{2}\left(g_{\beta}\left(y_{\alpha}\right)+h_{\alpha}\right)$, where $q_{1}, q_{2} \in \mathbb{Q}$ and $y \in \operatorname{rng}\left(f_{\alpha}^{(0)}+g_{\beta}\right)$. By (2) we obtain $q_{2}=0$. So $u_{\beta} \in$ $\operatorname{rng}\left(f_{\tau}+g_{\beta}\right)$, where $\tau=\min \left\{\gamma: u_{\beta} \in \operatorname{rng}\left(f_{\gamma}+g_{\beta}\right)\right\}$. By (iv), $\tau<\beta<\alpha$. But then $u_{\beta} \in \operatorname{rng}\left(f_{\tau}+g_{\beta}\right) \subset \operatorname{rng}\left(f_{\beta}+g_{\beta}\right)$, contrary to (iv).

Now set

$$
f_{\alpha}^{(1)}=L_{2}\left(f_{\alpha}^{(0)} \cup\left\{\left(y_{\alpha}, h_{\alpha}\right)\right\}\right) .
$$

If $h_{\alpha} \in \operatorname{dom}\left(f_{\alpha}^{(1)}\right)$, then put $f_{\alpha}=f_{\alpha}^{(1)}$. Otherwise choose

$$
v_{\alpha} \in H \backslash \bigcup_{\beta<\alpha}\left(L\left(\operatorname{rng}\left(f_{\alpha}^{(1)}+g_{\beta}\right) \cup\left\{u_{\beta}\right\}\right)-g_{\beta}\left(h_{\alpha}\right)\right)
$$

and put

$$
f_{\alpha}=L_{2}\left(f_{\alpha}^{(1)} \cup\left\{\left(h_{\alpha}, v_{\alpha}\right)\right\}\right)
$$

Finally, since card $\left(\operatorname{dom}\left(f_{\alpha}\right)\right)<2^{\omega}$, we can choose $u_{\alpha} \in H \backslash \operatorname{rng}\left(f_{\alpha}+g_{\alpha}\right)$. It can be easily seen that $f_{\alpha}$ fulfills conditions $(\mathrm{i})-(\mathrm{v})$.

Define $f$ by

$$
f=\bigcup_{\alpha<2^{\omega}} f_{\alpha}
$$

Because $H \subset \operatorname{dom}(f), f \in \mathcal{A} d d$. Since $\operatorname{ker}(f) \neq\{0\}$ and $H \subset \operatorname{rng}(f)$, by Lemma $1, f \in \mathcal{D}$. Notice that $f+g_{\beta} \notin \mathcal{D}$ for each $\beta<2^{\omega}$. Indeed, fix an arbitrary $\beta<2^{\omega}$. Then by (iv) $u_{\beta} \notin \operatorname{rng}\left(f+g_{\beta}\right)$. But, by (iii), $\operatorname{rng}\left(f+g_{\beta}\right)$ is dense in $\mathbb{R}$, which shows that $f+g_{\beta} \notin \mathcal{D}$.

Remark. Since all level sets of an additive function are congruent under translations, the condition $(\star)$ is equivalent to the following:

$(\star \star)$ the covering of the family $\mathcal{B}=\{\operatorname{ker}(g)+y: g \in \mathcal{G} \& y \in \mathbb{R}\}$ is equal to $2^{\omega}$. 
Corollary 1 Assume that $2^{\omega}$ is a regular cardinal, $\mathcal{G} \subset \mathcal{A d d}$, card $(\mathcal{G})=2^{\omega}$ and there exists an ideal $\mathcal{J} \supset\{\operatorname{ker}(g): g \in \mathcal{G}\}$ satisfies the following conditions:

(i) $\mathcal{J}$ is invariant under translations, i.e., $A+x \in \mathcal{J}$ for all $A \in \mathcal{J}$ and $x \in \mathbb{R}$

(ii) the covering of $\mathcal{J}$ is equal to $2^{\omega}$.

Then there is $f \in \mathcal{A} d d \cap \mathcal{D}$ such that $f+g \notin \mathcal{D}$ for each $g \in \mathcal{G}$.

If Martin's Axiom (MA) or the Continuum Hypothesis (CH) hold, then the ideals $\mathcal{K}$ of all meager sets and $\mathcal{N}$ of all null sets fulfill the statements (i) and (ii). Therefore we have the following corollary.

Corollary 2 (MA) Assume that $\mathcal{G}$ is a family of additive functions such that $\operatorname{card}(\mathcal{G})=2^{\omega}$ and either $\operatorname{ker}(g) \in \mathcal{N}$ for each $g \in \mathcal{G}$ or $\operatorname{ker}(g) \in \mathcal{K}$ for each $g \in \mathcal{G}$. Then there is $f \in \mathcal{A} d d \cap \mathcal{D}$ such that $f+g \notin \mathcal{D}$ for each $g \in \mathcal{G}$.

Proposition 1 The covering of the family $\mathcal{S}(\mathbb{R})$ of all proper linear subspaces of $\mathbb{R}$ over $\mathbb{Q}$ is equal to $\omega$.

Proof. Let $\mathcal{B}_{0} \subset \mathcal{S}(\mathbb{R})$ be such that $\bigcup \mathcal{B}_{0}=\mathbb{R}$. We will show that card $\left(\mathcal{B}_{0}\right) \geq$ $\omega$. By way of contradiction suppose that $\mathcal{B}_{0}=\left\{V_{1}, \ldots, V_{n}\right\}$ for some $n \in \mathbb{N}$. We may assume that $V_{i} \backslash \bigcup_{k \neq i} V_{k} \neq \emptyset$ for every $i \leq n$. Note that $n \geq 2$, because all $V_{i}$ are proper. For $i=1,2$ choose

$$
v_{i} \in V_{i} \backslash \bigcup_{k \neq i} V_{k}
$$

and set $v_{k}=(k-2) v_{2}+v_{1}$ for $k>2$ Then there exists $i \leq n$ for which the set $N_{i}=\left\{k: v_{k} \in V_{i}\right\}$ is infinite. Fix $j, k \in N_{i}$ with $2<j<k$. Then we have

$$
v_{k}-v_{j}=(k-j) v_{2} \in V_{2} \cap V_{i} .
$$

Therefore $v_{2} \in V_{i}$ and, by (3), $i=2$. But then $v_{1}=v_{k}-(k-2) v_{2} \in V_{2}$, contrary to the choice of $v_{1}$.

Now we will construct a family $\mathcal{B}_{0} \subset \mathcal{S}(\mathbb{R})$ such that card $\left(\mathcal{B}_{0}\right)=\omega$ and $\cup \mathcal{B}_{0}=\mathbb{R}$. Let $H \subset \mathbb{R}$ be an arbitrary Hamel basis and let $\left\{H_{n}: n \in \mathbb{N}\right\}$ be a partition of $H$ into proper subsets. Put

$$
\mathcal{B}_{0}=\left\{L\left(\bigcup_{n \in A} H_{n}\right): A \subset \mathbb{N}, \operatorname{card}(A)<\omega\right\}
$$

It is obvious that card $\left(\mathcal{B}_{0}\right)=\omega$ and $\mathbb{R} \notin \mathcal{B}_{0}$. Fix an arbitrary $x \in \mathbb{R}$. Then $x=\sum_{n=1}^{k} q_{n} h_{n}$ for some $k \in \mathbb{N}, q_{n} \in \mathbb{Q}$, and $h_{n} \in H n=1, \ldots k$. Since $A_{x}=\left\{j: \exists_{n \leq k} h_{n} \in H_{j}\right\}$ is finite, $x \in L\left(\bigcup_{n \in A_{x}} H_{n}\right) \in \mathcal{B}_{0}$. Consequently, $\cup \mathcal{B}_{0}=\mathbb{R}$. 
Proposition 2 If a family $\mathcal{G} \subset \mathcal{A} d d$ satisfies the condition $(\star)$, then $\operatorname{card}(\operatorname{rng}(g))=2^{\omega}$ for each $g \in \mathcal{G}$.

Proof. If card $(\operatorname{rng}(g))<2^{\omega}$ for some $g \in \mathcal{G}$, then $\bigcup_{y \in \operatorname{rng}(g)} g^{-1}(y)=\mathbb{R}$. So the covering of $\mathcal{G}$ is less than $2^{\omega}$.

Lemma 3 Assume that $n \in \mathbb{N}$ and $\mathcal{G}=\left\{g_{i}: i \leq n\right\}$ is a family of additive functions such that card $\left(\operatorname{rng}\left(g_{i}\right)\right)=2^{\omega}$ for $i \leq n$. Then $\mathcal{G}$ satisfies condition $(\star)$.

Proof. Let $\mathcal{B}_{0}$ be a subfamily of $\left\{g^{-1}(y): g \in \mathcal{G}\right.$ and $\left.y \in \mathbb{R}\right\}$ such that card $\left(\mathcal{B}_{0}\right)<2^{\omega}$. Define $Y_{i}=L\left(\left\{y \in \mathbb{R}: g_{i}^{-1}(y) \in \mathcal{B}_{0}\right\}\right)$ and $V_{i}=g_{i}^{-1}\left(Y_{i}\right)$. Note that card $\left(Y_{i}\right)<2^{\omega}$ for $i=1, \ldots, n$. Because card $\left(\operatorname{rng}\left(g_{i}\right)\right)=2^{\omega}, V_{i} \neq \mathbb{R}$ for every $i=1, \ldots, n$. So by Proposition $1, V=\bigcup_{i=1}^{n} V_{i} \neq \mathbb{R}$. But $\bigcup \mathcal{B}_{0} \subset V$. Thus $\bigcup \mathcal{B}_{0} \neq \mathbb{R}$, which completes the proof.

Lemma 4 Assume that $n \in \mathbb{N}, \mathcal{G}=\left\{g_{i}: i \leq n\right\} \subset \mathcal{A} d d$ and $\operatorname{card}(\operatorname{rng}(g))=$ $2^{\omega}$ for each $g \in \mathcal{G}$. Then there exists a linearly independent set $H_{1} \subset \mathbb{R}$ such that $\operatorname{card}\left(H_{1}\right)=2^{\omega}$ and $g_{i} \mid L\left(H_{1}\right)$ is an injection for each $i \leq n$.

Proof. Choose an arbitrary $h_{0} \neq 0$. Fix $\alpha<2^{\omega}$ and assume that we have chosen a linearly independent set $\left\{h_{\beta}: \beta<\alpha\right\}$ such that $g_{i} \mid L\left(\left\{h_{\beta}: \beta<\alpha\right\}\right)$ is an injection for each $i \leq n$. For each $i$ we have card $\left(L\left(\left\{g_{i}\left(h_{\beta}\right): \beta<\alpha\right\}\right)\right)<2^{\omega}$ and $\operatorname{card}\left(\operatorname{rng}\left(g_{i}\right)\right)=2^{\omega}$. So $g_{i}^{-1}\left(L\left(\left\{g_{i}\left(h_{\beta}\right): \beta<\alpha\right\}\right)\right)$ is a proper linear subspace of $\mathbb{R}$. By Theorem 1 , we obtain

$$
\mathbb{R} \backslash \bigcup_{i=1}^{n} g_{i}^{-1}\left(L\left(\left\{g_{i}\left(h_{\beta}\right): \beta<\alpha\right\}\right)\right) \neq \emptyset .
$$

Choose $h_{\alpha} \in \mathbb{R} \backslash \bigcup_{i=1}^{n} g_{i}^{-1}\left(L\left(\left\{g_{i}\left(h_{\beta}\right): \beta<\alpha\right\}\right)\right)$. Then the set $H_{1}=\left\{h_{\alpha}: \alpha<\right.$ $\left.2^{\omega}\right\}$ is linearly independent and $g_{i} \mid L\left(H_{1}\right)$ is an injection for $i=1, \ldots, n$.

Assuming $\operatorname{cf}\left(2^{\omega}\right)=2^{\omega}$, the next theorem is a consequence of Theorem 1 and Lemma 3. We shall prove it in ZFC, without additional set-theoretical assumptions.

Theorem 2 Assume that $\mathcal{G}=\left\{g_{i}: i=1, \ldots, n\right\} \subset \mathcal{A} d d$ and $\operatorname{card}(\operatorname{rng}(g))=$ $2^{\omega}$ for each $g \in \mathcal{G}$. Then there is $f \in \mathcal{A} d d \cap \mathcal{D}$ such that $f+g \notin \mathcal{D}$ for $g \in \mathcal{G}$.

Proof. Let $H_{1} \subset \mathbb{R}$ be the set constructed in Lemma 4 for the family $\mathcal{G}$ and let $H=\left\{h_{\alpha}: \alpha<2^{\omega}\right\} \supset H_{1}$ be a Hamel basis. Choose

$$
h \in H \backslash\left\{g_{i}\left(h_{2}\right): i=1, \leq n\right\}
$$


and set $f_{0}=L_{2}\left(\left\{\left(h_{0}, 0\right),\left(h_{1}, h_{0}\right),\left(h_{2}, h\right)\right\}\right)$. Clearly card $\left(\operatorname{rng}\left(f_{0}+g_{i}\right)\right)=\omega$ for $i=1, \ldots, n$.

For $i=1, \ldots, n$ choose $u_{i} \in H \backslash \operatorname{rng}\left(f_{0}+g_{i}\right)$. For every $0<\alpha<2^{\omega}$ we will construct an additive function $f_{\alpha}$ such that

(i) $f_{0} \subset f_{\beta} \subset f_{\alpha}$ for $\beta<\alpha$,

(ii) $h_{\alpha} \in \operatorname{dom}\left(f_{\alpha}\right) \cap \operatorname{rng}\left(f_{\alpha}\right)$,

(iii) $u_{i} \notin \operatorname{rng}\left(f_{\alpha}+g_{i}\right)$ for each $i=1,2, \ldots, n$,

(iv) $\operatorname{card}\left(\operatorname{dom}\left(f_{\alpha}\right)\right)=\max (\omega, \alpha)$.

Fix $\alpha<2^{\omega}$ and assume that for $\beta<\alpha$ we have chosen the function $f_{\beta}$ which satisfies conditions (i)-(iv). Set

$$
f_{\alpha}^{(0)}=\bigcup_{\beta<\alpha} f_{\beta} .
$$

We consider two cases. If $h_{\alpha} \in \operatorname{rng}\left(f_{\alpha}^{(0)}\right)$, then define $f_{\alpha}^{(1)}=f_{\alpha}^{(0)}$. Otherwise we will choose $y_{\alpha} \in H$ such that $y_{\alpha} \notin \operatorname{dom}\left(f_{\alpha}^{(0)}\right)$ and

$$
u_{i} \notin L\left(\operatorname{rng}\left(f_{\alpha}^{(0)}+g_{i}\right) \cup\left\{g_{i}\left(y_{\alpha}\right)+h_{\alpha}\right\}\right) \text { for } i \leq n .
$$

To choose $y_{\alpha}$, observe that $g_{i} \mid H_{1}$ is an injection, and by (iv),

$$
\operatorname{card}\left(L\left(\operatorname{rng}\left(f_{\alpha}^{(0)}+g_{i}\right)\left\{u_{i}\right\}\right)-h_{\alpha}\right)<2^{\omega} .
$$

So the cardinality of the set

$$
A_{\alpha, i}=\left(g_{i} \mid H_{1}\right)^{-1}\left(L\left(\operatorname{rng}\left(f_{\alpha}^{(0)}+g_{i}\right)\left\{u_{i}\right\}\right)-h_{\alpha}\right)
$$

is less than $2^{\omega}$. Therefore, the cardinality of $A_{\alpha}=H_{1} \backslash \bigcup_{i=1}^{n} A_{\alpha, i}$ is equal to $2^{\omega}$. Take an arbitrary $y_{\alpha} \in A_{\alpha}$. Now the proof of (6) is analogous to the proof of condition (1) in Theorem 1.

Next let $f_{\alpha}^{(1)}=L_{2}\left(f_{\alpha}^{(0)} \cup\left\{\left(y_{\alpha}, h_{\alpha}\right)\right\}\right)$. If $h_{\alpha} \in \operatorname{dom}\left(f_{\alpha}^{(1)}\right)$, then put $f_{\alpha}=$ $f_{\alpha}^{(1)}$. Otherwise choose

$$
v_{\alpha} \in H \backslash \bigcup_{i=1}^{n}\left[L\left(\operatorname{rng}\left(f_{\alpha}^{(1)}+g_{i}\right) \cup\left\{u_{i}\right\}\right)-g_{i}\left(h_{\alpha}\right)\right]
$$

and let $f_{\alpha}=L_{2}\left(f_{\alpha}^{(1)} \cup\left\{\left(h_{\alpha}, v_{\alpha}\right)\right\}\right)$. It can be seen that $f_{\alpha}$ fulfills conditions (i)-(iv). 
Finally, let $f=\bigcup_{\alpha<2^{\omega}} f_{\alpha}$. Because $H \subset \operatorname{dom}(f), f \in \mathcal{A} d d$. Since $\operatorname{ker}(f) \neq$ $\{0\}$ and $H \subset \operatorname{rng}(f)$, by Lemma $1, f \in \mathcal{D}$.

Notice that $f+g_{i} \notin \mathcal{D}$ for each $i=1, \ldots, n$. Indeed, fix arbitrary $i \leq n$. Then by (iii) $u_{i} \notin \operatorname{rng}\left(f+g_{i}\right)$. But conditions (4) and (i) imply that rng $\left(f+g_{i}\right)$ is dense in $\mathbb{R}$. Thus $f+g_{i} \notin \mathcal{D}$.

Corollary $3 \mathcal{M}_{a}(\mathcal{A} d d \cap \mathcal{D})=\{0\}$.

Proof. The inclusion " $\supset$ " is obvious. To prove the inclusion " $\subset$ " assume that $f \in \mathcal{M}_{a}(\mathcal{A} d d \cap \mathcal{D}) \backslash\{0\}$. Note that $f \in \mathcal{A} d d \cap \mathcal{D}$, because the constant function $g \equiv 0$ belongs to the class $\mathcal{A} d d \cap \mathcal{D}$. So, $\operatorname{rng}(f)=\mathbb{R}$. By Theorem 2, $f+h \notin \mathcal{A} d d \cap \mathcal{D}$ for some $h \in \mathcal{A} d d \cap \mathcal{D}$. Hence $f \notin \mathcal{M}_{a}(\mathcal{A} d d \cap \mathcal{D})$, an impossibility.

The importance of the assumptions in Theorem 1 is not clear. In particular, the following problem is open.

Problem 1 Assume $C H$ and $\mathcal{G}=\left\{g_{\alpha}: \alpha<2^{\omega}\right\}$ is a family of additive functions. Does there exists $f \in \mathcal{A} d d \cap \mathcal{D}$ such that $f+g_{\alpha} \notin \mathcal{D}$ for each $\alpha$ ?

\section{References}

[1] A. M. Bruckner and J. Ceder, On the sum of Darboux functions, Proc. Amer. Math. Soc. 51 (1975), 97-102.

[2] A. L. Cauchy, Cours d'analyse de l'Ecole Polytechnique, 1. Analyse algébrique, V., Paris, 1821.

[3] B. Kirchheim and T. Natkaniec, On universally bad Darboux functions, Real Analalysis Exch. 16 (1990-1991), 481-486.

[4] P. Komjáth, A note on Darboux functions, Real Analalysis Exch. 18 (1992-93), 249-252.

[5] M. Kuczma, An introduction to the theory of functional equations and inequalities. Cauchy's equation and Jensen's inequality, PWN WarszawaKraków-Katowice 1985.

[6] T. Radaković, Über Darbouxsche und stetige Funktionen, Monat. Math. Phys. 38 (1931), 117-122.

[7] J. Steprans, Sums of Darboux and continuous functions, Fund. Math. 146 (1995), 107-120.

[8] R. Švarc, On the range of values of the sum of a continuous and a Darboux function, Čas. Pest. Mat. 98 (1973), 178-180, 213. 